\title{
A New AMS Journal Launching in 2021
}

\section{Ralph L. Cohen and Qiang Du}

In 2021, the American Mathematical Society is launching a major new electronic-only, diamond open access journal: Communications of the AMS (CAMS). CAMS is designed as a research journal that sits at the interface of theoretical and applied mathematics, embracing the highest quality mathematical research with a holistic sense of the future of mathematics.

The two of us are the Managing Editors of CAMS. When we first heard that the AMS was considering launching a new journal, both of our initial reactions were, "Why is there a need for a new journal?" We knew that the goal was for it to be a top-level journal, which we thought was great. But on the other hand, there are many high-quality journals in which top-level research can be published. After many conversations with many mathematicians, we came away with the view that there are at least two compelling reasons for the AMS to establish and support this new journal.

One reason is the need for high-quality open-access publications. CAMS will be open access done the way it should be done: as a diamond open access journal, CAMS will be free to both the authors and the readers. CAMS will be a first-rate journal that is freely and readily available to the entire mathematical community. This is something the AMS can and should be proud of producing, and something the entire mathematical community can be proud to support.

Ralph L. Cohen is the Barbara Kimball Browning Professor Emeritus in the School of Humanities and Sciences and professor of mathematics at Stanford University. His email address is r1c@stanford.edu.

Qiang Du is the Fu Foundation Professor of Applied Mathematics in the Department of Applied Physics and Applied Mathematics, Fu Foundation School of Engineering and Applied Science, at Columbia University. His email address is qd2125@columbia.edu.

For permission to reprint this article, please contact: reprint-permission @ams.org.

DOI: https://dx.doi.org/10.1090/noti2376
The other reason is mathematical. CAMS will be a journal publishing mathematics of the highest level across the full spectrum of mathematics. It will publish papers in "pure mathematics" that prove theorems, solve problems, resolve conjectures, or develop new theories or techniques. It will also publish papers in "applied mathematics" that motivate new mathematical problems and stimulate new mathematical concepts, or use mathematics to address questions arising from a multitude of disciplines of science and technology. It is so important for the AMS to publish a journal that represents this breadth now because in recent years, the boundary between pure and applied mathematics has been blurred to the point of being obliterated.

Some of the most theoretical areas of mathematics are now finding unexpected applications. For example, techniques from algebraic topology are now being used in data analysis, with applications to such diverse areas as cancer research, imaging, and neuroscience. Number theory and algebraic geometry continue to be used in coding theory as well as other applications to cybersecurity. Conversely, tremendous growth in many mathematical disciplines has been fueled by technological advances and challenges rooted not only in traditional subjects like physics but also biology, materials science, and computer and information sciences, with signal processing, social networks, and machine learning as good recent examples. New conjectures in mathematics are being motivated from applications and are being generated with the help of new algorithms and computational experiments, and resulting theorems are being proved.

There has never been a time when mathematics was at once so broad, both in its theory and in its applications, and also so connected. As a society that represents the interests of the global mathematical community, it is highly appropriate for the AMS to publish this new, top-level, diamond open access journal that promotes equal free access with 
broad and diverse representation, benefiting the entirety of the mathematics profession.

Joining us in realizing the vision of CAMS are a stellar group of senior editors:

- Douglas Arnold (University of Minnesota)

- Ingrid Daubechies (Duke University)

- Bjorn Engquist (University of Texas)

- Irene Fonseca (Carnegie Mellon University)

- Daniel Freed (University of Texas, Austin)

- Martin Hairer (Imperial College)

- Robert Lazarsfeld (Stony Brook University)

- Andras Vasy (Stanford University)

- Gigliola Staffilani (Massachusetts Institute of Technology)

- Amie Wilkinson (University of Chicago)

and 20 other leading mathematicians representing all fields, acting as associate editors:

- Ian Agol (University of California, Berkeley)

- Bhargav Bhatt (University of Michigan)

- Liliana Borcea (University of Michigan)

- Romain Dujardin (Université Pierre et Marie Curie)

- Marco Gualtieri (University of Toronto)

- C. Sinan Gunturk (New York University)

- Martin J. Gander (University of Geneva)

- Ben Green (University of Oxford)

- Kathryn P. Hess (École polytechnique fédérale de Lausanne)

- Inwon Kim (University of California, Los Angeles)

- Jianfeng Lu (Duke University)

- Kathryn Mann (Cornell University)

- Siddhartha Mishra (ETH Zurich)

- Kavita Ramanan (Brown University)

- Nick Sheridan (University of Edinburgh)

- Joel A. Tropp (California Institute of Technology)

- Chelsea Walton (Rice University)

- Lauren Williams (Harvard University)

- Juncheng Wei (University of British Columbia)

- Wei Zhang (Massachusetts Institute of Technology)

This is just the beginning of the story for Communications of the AMS, and we encourage you to submit to the journal and read papers as they appear.

Learn more about CAMS, including how to submit articles for review, at https://www. ams.org/cams.

\section{Embracing Openness in Research and Publishing}

As the global research ecosystem inexorably moves towards openness, how should the AMS respond in a way that suits mathematicians across the world? In the development of Communications of the American Mathematical Society, we sought to create an open model for a new journal that had no fees to publish and was free to read, yet still had a source of funds to do so in a sustainable way. In addition, to be truly open access required appropriate reuse terms.

The first piece of the puzzle came from a generous anonymous donor to the AMS. The donor is funding the launch of CAMS and will create an endowed fund to ensure its continued publication of in perpetuity.

Secondly, the journal will allow the author to retain copyright, offering two Creative Commons reuse licenses. Under a CC BY license, an article is free to reuse in any setting with appropriate author attribution. Under a CC BY NC license, an article is free to reuse in a non-commercial setting with appropriate author attribution. The choice of reuse license will sit with the author.

We are thrilled to launch Communications of the AMS: the first broad-based, diamond open access journal that reaches across pure and applied mathematics. The leadership of Communications of the AMS reflects the broad appeal of the journal to authors and readers and clearly sets a standard for the quality of articles that will be published.

\section{—Robert Harington, AMS Associate Executive Director} and Head of Publishing Division 